\title{
Spontaneous Nasal Septal Abscess Presenting as Complete Nasal Obstruction
}

\author{
Joseph Chun-Kit Chung ${ }^{*}$, Athena Ting-Ka Wong, Wai-Kuen Ho \\ Division of Otorhinolaryngology, Head \& Neck Surgery, Department of Surgery, The University of Hong Kong, \\ Queen Mary Hospital, Hong Kong, China

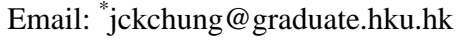

Received March 23, 2013; revised April 3, 2013; accepted May 3, 2013

Copyright (C 2013 Joseph Chun-kit Chung et al. This is an open access article distributed under the Creative Commons Attribution License, which permits unrestricted use, distribution, and reproduction in any medium, provided the original work is properly cited.

\begin{abstract}
Nasal septal abscess is an uncommon condition, yet presents as a rhinological emergency. Its symptoms resemble upper respiratory tract infection and the diagnosis may be missed leading to intracranial complication and cosmetic deformity. We present a healthy patient with idiopathic nasal septal abscess who complained of acute complete nasal obstruction, fever and nasal pain. Common aetiologies, causative agents, complications and management of nasal septal abscess are discussed.
\end{abstract}

Keywords: Nasal Septum; Abscess; Emergencies

\section{Introduction}

Nasal septal abscess is an uncommon condition. High index of suspicion and prompt drainage is required to prevent intracranial infection and future nasal deformity. However the clinical manifestations may be subtle and mimic upper respiratory tract infection. It usually happens after surgery or trauma. Here we present a case of spontaneous nasal septal abscess and discuss the management plan.

\section{Case Report}

A 41-year-old gentleman who enjoyed good past health was referred to our ENT clinic by his family physician with four days history of complete nasal obstruction, fever and nasal pain. He also had prior history of myalgia and headache for 1 week. There was no prior history of nasal surgery, trauma. On physical examination, his nasal dorsum was swollen and tender. Anterior rhinoscopy revealed bilateral cherry red septal bulge (Figure 1). Other than running a fever of $38.8^{\circ} \mathrm{C}$, there was no associated neurological deficit or neck stiffness. The rest of the examination including nasoendoscopy was unremarkable. The diagnosis of nasal septal abscess was confirmed by needle aspiration of pus. The sample was sent for culture and sensitivity testing. His white blood cell count was elevated to $2.1 \times 10^{10} / 1$ with neutrophil pre-

${ }^{*}$ Corresponding author. dominance. Blood glucose was normal. Urgent CT scan revealed a $3 \mathrm{~cm} \times 1.2 \mathrm{~cm} \times 1.6 \mathrm{~cm}$ ill-defined rim enchancing hypodense collection at the anterior nasal septum (Figures 2(a) and (b)). The rest of the paranasal sinuses were clear. Dental assessment later could not identify any infection of dental origin.

Emergency transnasal drainage of the abscess under general anaesthesia was subsequently performed. Intraoperatively, the central portion of cartilaginous nasal septum was necrotic and destroyed by infection. The superior and caudal septal cartilage struts were still intact, but soften and thinned as a result of inflammation (Figure 3). A drain was anchored in the abscess cavity and both nasal cavities were packed with merocele.

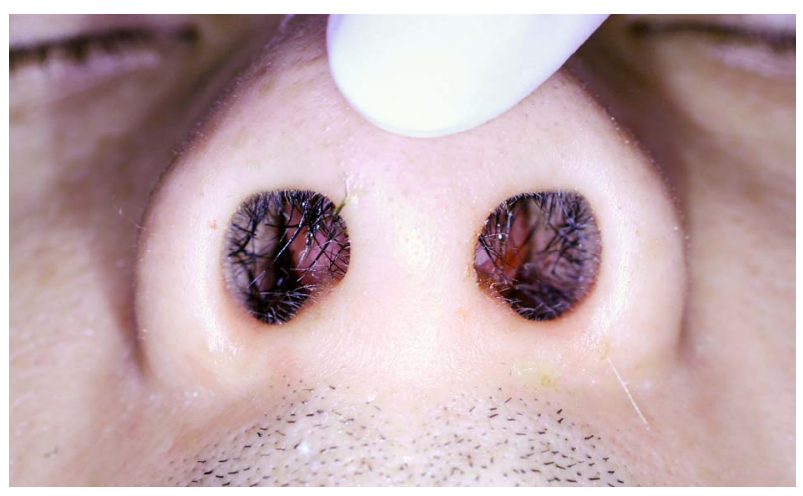

Figure 1. Nasal septal abscess resembling hypertrophic turbinates. 


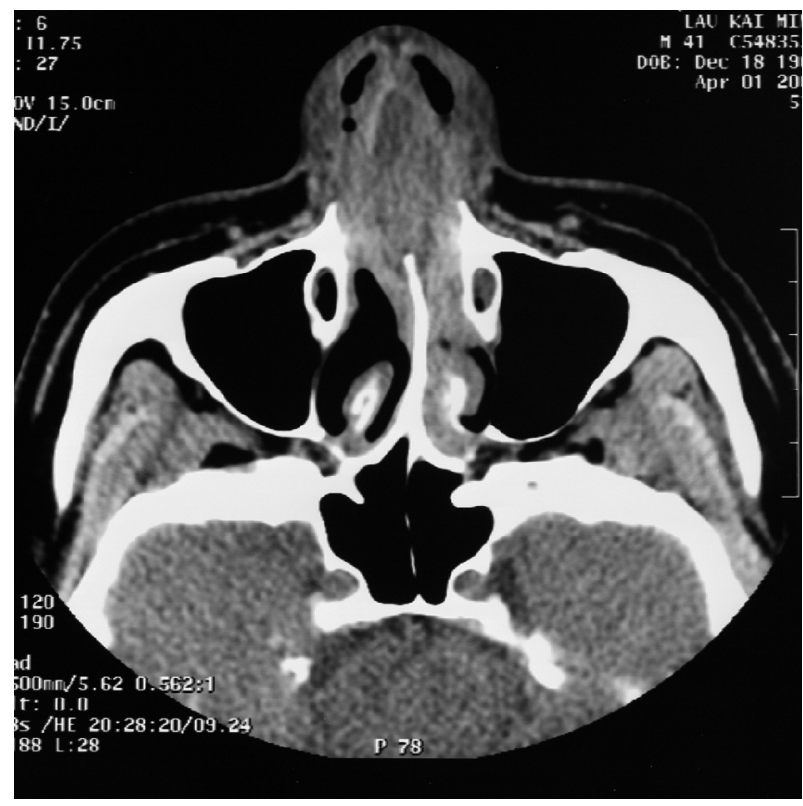

(a)

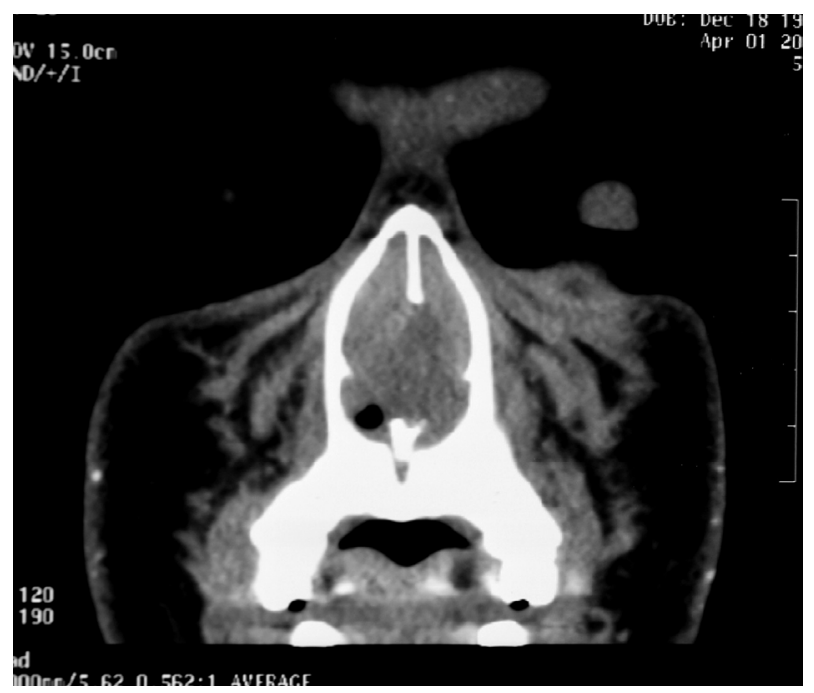

(b)

Figure 2. Computer tomographic scan: (a) Axial cut showing abscess involving anterior cartilaginous nasal septum; (b) Coronal cut showing showing no intra-cranial extension.

Bacteriological culture yielded methicillin-sensitive Staphylococcus aureus that was sensitive to Augmentin. Patient was treated accordingly for 2 weeks. Follow up nasoendoscopy at 2 weeks showed intact nasal septum and complete resolution of the abscess. At 6 months later, he noted a mild depression over his nasal dorsum. Augmentation rhinoplasty has been suggested, but he refused.

\section{Discussion}

Nasal septal abscess is a collection of pus between the nasal septal cartilage or bony septum and the mucoperichondrium or mucoperostium [1]. This entity was first

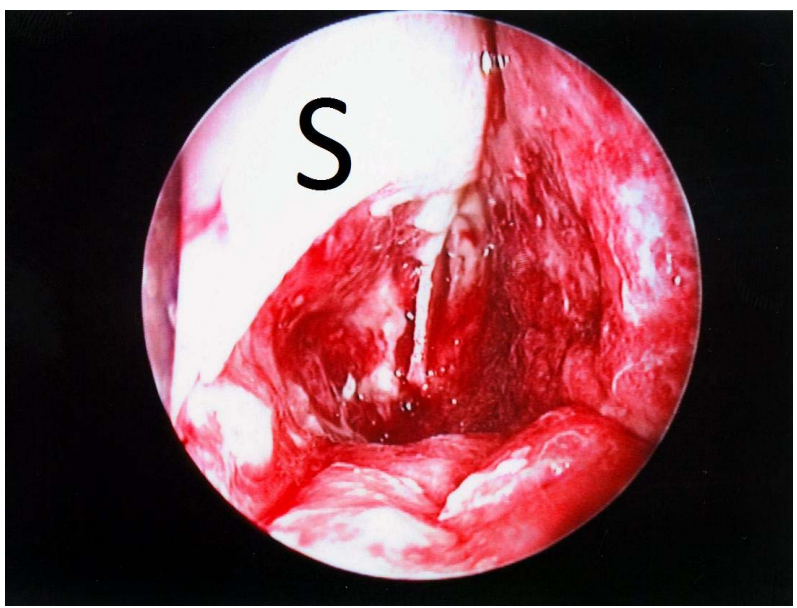

Figure 3. Central cartilage destruction by inflammation, superior and caudal strut (S) still preserved.

reported in 1810 by Arnal who assisted Cloquet to drain a nasal septal abscess in a patient suffering from "coryza" [2]. The commonest aetiology is nasal trauma leading to haematoma formation and subsequent infection [2,3]. Nearly $75 \%$ are secondary to nasal injury [1]; less frequently following septal surgery. Other causes include localized nasal sinusitis, vestibulitis [3-5]; nearby dental abscess, infected dentigerous cyst [6]; an immunocompromised state in patients who suffered from diabetes mellitus, HIV infection or receiving chemotherapy [2,7]. In the literature, there are only two idiopathic nasal septal abscesses reported previously [8], similar to the present case. Most of the abscess cavity situated at the anterior cartilaginous nasal septum. Posterior septal abscess may be missed if only anterior rhinoscopy is performed [4].

The most common presenting symptom of nasal septal abscess is nasal obstruction and pain [2], in distinction with uncomplicated septal haematoma which usually presents as painless nasal obstruction after injury. Other symptoms include fever, malaise, headache and epistaxis. On rhinoscopy, this uncommon pathology is often mistaken as inferior turbinate hypertrophy, deviated nasal septum or simple mucosal oedema $[2,4]$ by less experience physicians and causal examination. This may be avoided by cautious inspection and palpation, confirming a fluctuant swelling arises from nasal septum.

The accumulation of pus between the cartilage and perichondrium will lead to ischaemia and pressure necrosis of the cartilage. Together with the digestive process of leukocytes and Cathepsin D, an enzyme responsible for reshaping the quadrangular cartilage, this may result in septal cartilage destruction, saddle nose deformity and lead to both functional and cosmetic problems [9]. In a growing child in particular, there may be additional disturbance of the normal development of the nose and maxilla $[9,10]$. Delayed diagnosis and management may also lead to life-threatening intracranial infective com- 
plications such as brain abscess, meningitis and cavernous sinus thrombosis, especially in immunocompromised patients [2-8].

Prompt recognition with surgical drainage of nasal septal abscess and antibiotic administration is thus required. The commonest aetiological agent is Staphylococcus aureus [3], others include Haemophilus influenzae, Streptococcus pneumonia and group A beta-haemolytic streptococcus [5]. In immunocompromised patients, the abscess may be caused by anaerobes or polymicrobial infections. Opportunistic fungal agents, for instance Candida, Cryptococcus and Aspergillus have been reported in HIV or poorly controlled DM patients resulting in a high mortality [2,7]. With this knowledge of microbiology, together with the general condition of the patient, empirical antibiotic treatment can be started immediately once diagnosis is made before the organism is isolated and its sensitivity is identified.

In case of nasal deformity after complete or near complete septal destruction, reconstruction of the nasal septum may be performed to address both functional and cosmetic problems. It may be carried out immediately after drainage of the abscess as a primary treatment, or secondary treatment after resolution of the infection $[6,9]$. Reconstruction of the destroyed septal infrastructure may be made use of residual septal cartilage by mosaicplasty or exchange technique; or autologous cartilage grafts from tragus, auricle or rib $[9,10]$.

In conclusion, non-traumatic nasal septal abscess is a rarely seen rhinological emergency. High index of suspicion and careful examination is essential because of its non specific flu-like symptoms. Early drainage would prevent nasal deformity and intra-cranial complications.

\section{REFERENCES}

[1] P. S. Ambrus, R. D. Eavey, A. S. Baker, W. R. Wilson and J. H. Kelly, "Management of Nasal Septal Abscess," Laryngoscope, Vol. 91, No. 4, 1981, pp. 575-582. doi:10.1288/00005537-198104000-00010 doi:10.1288/00005537-198104000-00010

[2] S. B. Shah, A. H. Murr and K. C. Lee, "Nontraumatic Nasal Septal Abscesses in the Immunocompromised: Aetiology, Recognition, Treatment and Sequelae,” American Journal of Rhinology, Vol. 14, No. 1, 2000, pp. 39-43. doi:10.2500/105065800781602975

[3] M. A. B. Jalaludin, "Nasal Septal Abscess-Retrospective Analysis of 14 Cases from University Hospital, Kuala Lumpur,” Singapore Medical Journal, Vol. 34, No. 5, 1993, pp. 435-437.

[4] A. George, W. K. Smith, S. Kumar and A. G. Pfleiderer, "Posterior Nasal Septal Abscess in a Healthy Adult Patient,” Journal of Laryngology \& Otology, Vol. 122, No. 12, 2008, pp. 1386-1388. doi:10.1017/S0022215107000886

[5] P. H. Huang, Y. C. Chiang, T. H. Yang, P. Z. Chao and F. P. Lee, "Nasal Septal Abscess," Otolaryngology_Head \& Neck Surgery, Vol. 135, No. 2, 2006, pp. 335-336. doi:10.1016/j.otohns.2005.09.015

[6] J. G. Cho, H. W. Lim, P. Zodpe, H. J. Kang and H. M. Lee, "Nasal Septal Abscess: An Unusual Presentation of Dentigerous Cyst,” European Archives of Otorhinolaryngology, Vol. 263, No. 11, 2006, pp. 1048-1050. doi:10.1007/s00405-006-0105-z

[7] R. Walker, L. Gardner, R. Sindwani, "Fungal Nasal Septal Abscess in the Immunocompromised Patient," Otolaryngology-Head \& Neck Surgery, Vol. 136, No. 3, 2007, pp. 506-507. doi:10.1016/j.otohns.2006.07.022

[8] B. Salam and A. Camilleri, "Non-Traumatic Nasal Septal Abscess in an Immunocompetent Patient," Rhinology, Vol. 47, No. 4, 2009, pp. 476-477.

[9] C. Dispenza, C. Saraniti, F. Dispenza, C. Caramanna and F. A. Salzano, "Management of Nasal Septal Abscess in Childhood: Our Experience,” International Journal of Pediatric Otorhinolaryngology, Vol. 68, No. 11, 2004, pp. 1417-1421. doi:10.1016/j.ijporl.2004.05.014

[10] D. J. Menger, I. C. Tabink and G. J. Trenite, "Nasal Septal Abscess in Children: Reconstruction with Autologous Cartilage Grafts on Polydioxanone Plate," Archives of Otolaryngology—Head \& Neck Surgery, Vol. 134, No. 8, 2008, pp. 842-847. doi:10.1001/archotol.134.8.842 